\title{
The efficacy and safety of cilostazol for the secondary prevention of ischemic stroke in acute and chronic phases in Asian population- an updated meta-analysis
}

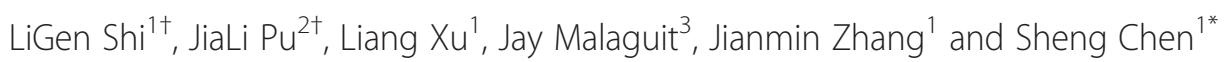

\begin{abstract}
Backgrounds: While previous meta-analysis have investigated the efficacy of cilostazol in the secondary prevention of ischemic stroke, they were criticized for their methodology, which confused the acute and chronic phases of stroke. We present a new systematic review, which differs from previous meta-analysis by distinguishing between the different phases of stroke, and includes two new randomized, controlled trials (RCTs).

Methods: All RCTs investigating the effect of cilostazol on secondary prevention of ischemic stroke were obtained. Outcomes were analyzed by Review Manager, including recurrence of cerebral infarction (ROCl), hemorrhage stroke or subarachnoid hemorrhage (HSSH), all-cause death (ACD), and modified Rankin Scale score (mRS). The Grading of Recommendations Assessment, Development and Evaluation (GRADE) assessed the quality of the evidence.

Results: 5491 patients from six studies were included in the current study. In secondary prevention of ischemic stroke in chronic phase, cilostazol was associated with a $47 \%$ reduction in ROCI (relative risk [RR] 0.53, 95\% confidence interval $[\mathrm{Cl}] 0.34$ to $0.81, p=0.003$ ), while no significant difference in $\mathrm{HSSH}$ and ACD compared with placebo; and $71 \%$ reduction in HSSH (RR 0.29, 95\% Cl 0.15 to $0.56, p=0.0002$ ) compared with aspirin, but not in $\mathrm{ROCl}$ and $\mathrm{ACD}$. In the secondary prevention of ischemic stroke in acute phase, cilostazol did not show any effect in the ROCI, HSSH, ACD and mRS compared to placebo or aspirin. The quality of the evidence from chronic phase was high or moderate, and those from acute phase were moderate or low when analyzed by GRADE approach.
\end{abstract}

Conclusion: Cilostazol provided a protective effect in the secondary prevention of the chronic phase of ischemic stroke.

Keywords: Acute Phase, Chronic phase, Cilostazol, Meta-analysis, Stroke

\section{Background}

Stroke accounts for $10 \%$ of all deaths worldwide [1], and is the second leading cause of mortality in China [2]. Recurring strokes in approximately $30 \%$ of patients showed more severe consequences than primary stroke, usually leading to dementia and death [3]. Thus, secondary prevention in high-risk patients with a previous stroke becomes extremely important. Among the various medical

\footnotetext{
* Correspondence: saintchan@sina.com

${ }^{\dagger}$ Equal contributors

'Department of Neurosurgery, Second Affiliated Hospital, School of Medicine, Zhejiang University, 88 Jiefang Road, Hangzhou 310009, Zhejiang, China
} Full list of author information is available at the end of the article managements, aspirin plays a pivotal role in the secondary prevention of stroke because of its antiplatelet efficacy [4]. However, this efficacy has prominent raceethnic differences, a recent study reported that Asians have a higher risk of recurrent ischemic and hemorrhagic stroke in the secondary stroke prevention phase [5]. Besides systemic hemorrhagic events, common side effects including dyspepsia, abdominal pain, and gastric ulcers have also limited its clinical application [6,7]. It is therefore necessary to develop an effective new drug with a lower incidence of side effects for aspirin intolerant populations. Cilostazol, an antiplatelet agent with selectively inhibiting phosphodiesterase III, is such an 
agent [8]. It not only prevents the inactivation of intracellular cyclic adenosine monophosphate (cAMP) and inhibits platelet aggregation, but also improves endothelial function and inhibits the proliferation of arterial smooth muscle cells [9]. Furthermore, several randomized controlled trials (RCTs) indicate that cilostazol had lessintracranial hemorrhage risks, compared with aspirin in the secondary prevention of the stroke $[2,4,9,10]$.

Previous systematic reviews and meta-analysis of RCTs determined whether cilostazol reduces morbidity and mortality compared with aspirin for the secondary prevention of stroke $[11,12]$. However, these meta-analysis were incomplete in several respects. The major criticism was its methodology that confused the acute and chronic phases of stroke. It has been confirmed that the acute phase of reperfusion injury exists in animal models of ischemic stroke, which plays an important role in the microcirculation levels [13]. It has also been confirmed that $20 \%$ to $40 \%$ of all patients in acute phase have aprogressive worsening of clinical and/or radiologic features even with currently available treatments [14]. Furthermore, recent studies have demonstrated that cilostazol might be a protective agent in the secondary prevention of the chronic phases of stroke $[2,4,9,15]$. But the benefit of cilostazol in the prevention of stroke was controversial $[10,16]$. The minor criticisms contributing to the unsoundness of previous meta-analysis included the lack of data pertaining to the efficacy of cilostazol compared with a placebo. A recent RCT reported negative results of cilostazol against acute progressing stroke [16], which differed from a previous RCT [10] and might reverse the conclusion of the previous meta-analysis. Hence, we present a new systematic review, which differs from the previous systematic reviews in their methodology and inclusion of another two new RCTs. Moreover, the Grading of Recommendations Assessment, Development and Evaluation (GRADE) approach was applied to assess the quality of the evidence [17-19].

\section{Methods}

\section{Study protocol}

At the beginning of this project, a study protocol was drafted following the Cochrane Collaboration format [20].

\section{Eligibility criteria}

The present systematic review only included studies which met the following criteria: 1) study type: RCTs; 2) language restriction: only English studies were reviewed; 3) participants: adult patients suffered ischemic stroke; 3) intervention: cilostazol; 4) comparator: aspirin or placebo; 5) outcomes: recurrence of cerebral infarction (ROCI), hemorrhage stroke or subarachnoid hemorrhage (HSSH), and all-cause death (ACD). Exclusion criteria: 1) study types: case control study, cohort study, and retrospective study; 2) withdraw rate: $>20 \%$; 3) participants: $<18$ years.

\section{Search strategy and information sources}

Two of the authors (LGS and JLP) independently searched the Medline database up to March 2014 for the combination of the variables "cilostazol" AND "stroke". The search was limited to clinical studies and matched the titles and abstracts of studies. Moreover, we searched for all relevant RCT or meta-analysis studies in the Embase, Cochrane Library and the Cochrane Central Register of Controlled Trials published between Jan 1980 and March 2014. To insure all relevant studies had been included in this systematic review, besides the electronic database search, reference lists from RCTs and systematic reviews were manually screened. The appendices include details of the search strategies (Appendix).

\section{Study selection and data collection}

We included RCTs that assessed the efficacy and acceptability of cilostazol compared with placebo or aspirin treatment in patients with the history of stroke, as diagnosed by computed tomography (CT) or magnetic resonance imaging (MRI). All patients were recruited with the results for the secondary prevention of ischemic stroke in acute and chronic phases as separate subgroups. The acute phase of ischemic stroke was diagnosed when the patient suffered a cerebral infarction within 48 hours prior to participation in the trial without serious complications, and the chronic phase was defined from 1 to 6 months. The outcomes included as the following: ROCI, HSSH, ACD, and modified Rankin Scale score (mRS), all based on intention-to-treat datasets.

After reading all included RCT articles, we extracted the following data, which were described in all studies: country, single or multiple therapeutic centers, inclusion criteria for the participants, general information of the patients (age and gender), and therapeutic schedule (usage of the drugs, doses, and duration). The four outcomes were also selected from each trial.

\section{Risk of bias and quality assessment}

The Cochrane Collaboration tool was used in this systematic review to assess the risk of bias in each included RCT study. Two review authors (LGS and JLP) were independently assessed for methodological quality by the following six items: random sequence generation and allocation concealment (selection bias), blinding of participants and personnel (performance bias), blinding of outcome assessment (detection bias), incomplete outcome data (attrition bias), selective reporting (reporting bias) and other potential biases. For each item, the table provides a description and judgment rated as "low", "unclear" or "high" risk of bias. The risk of bias plot 
was created using the Review Manager 5.2 software. GRADE approach was used to assess the quality of the evidence. In this approach, we mainly assessed five items, including risk of bias, inconsistency, indirectness, imprecision and publication bias, which can affect the quality of evidence. For each item, the table provides a judgment criteria rated as "high, moderate, low or very low". After assessment of the evidence, GRADE pro 3.6 software created an evidence profiletable.

\section{Summary measures and synthesis of results}

Data was processed in Review Manager 5.2 from the Cochrane Collaboration. Dichotomous outcomes were analyzed as the risk ratio (RR; 95\% confidence interval [CI]) using the Mantel-Haenszel technique and a fixed effect model. Statistical heterogeneity was estimated by the $I^{2}$ statistic as follows: $I^{2}<30 \%$ means "low heterogeneity", $I^{2}=30 \%$ to $50 \%$ denotes "moderate heterogeneity", and $I^{2}>50 \%$ represents "substantial heterogeneity". Tests were two-tailed and a $p$ value less than 0.05 was considered to be significant for all analysis.

\section{Results}

We retrieved 763 records after the initial search strategy that scanned for title and abstract. A further 753 records were excluded either for unrelated to the study question or not a RCT, resulting in 10 papers for further assessment. Another 4 records have been excluded from this analysis for the following reasons: meta-analysis record, cilostazol and aspirin combined therapy, irrelevant outcomes, and duplicate data. Finally, six RCTs on the basis of the inclusion criteria (Figure 1) were included with a total of 5491 patients.

\section{Study characteristics}

The main characteristics and outcome events of the included RCT studies are listed in Table 1. The six RCTs, combined, enrolled 5491 patients including 965 patients in acute phase and 4526 patients in chronic phase of ischemic stroke. All the patients come from an Asian background, such as Japan, China, and Korea. The age of patients ranged from 59.4 to 66.6 years old. The follow-up time for the acute phase of ischemic stroke was 3 months, with the follow-up time for the chronic phase ranging from 1 to 5 years.

\section{Outcomes analysis}

For all analysis pertaining to efficacy and acceptability, no evidence exists for the between-study of heterogeneities assessed by Cochrane $I^{2}$ statistic (data not shown). No significant publication bias was shown in the funnel plots (data not shown).

\section{The efficacy and safety of cilostazol in chronic phase}

Results for this analysis and the quality of this evidence were presented in Table 2. For the analysis of the efficacy and safety of cilostazol in chronic phase, all 4526 patients from all 4 studies were available (1067 patients from 1 study randomized to cilostazol or placebo, and 3459 patients from 3 studies to cilostazol or aspirin). In the placebo-controlled study, cilostazol therapy reduced the ROCI by $47 \%$ (RR $0.53,95 \%$ CI 0.34 to 0.81 , $p=0.003$ ), and showed similar incidence in the HSSH

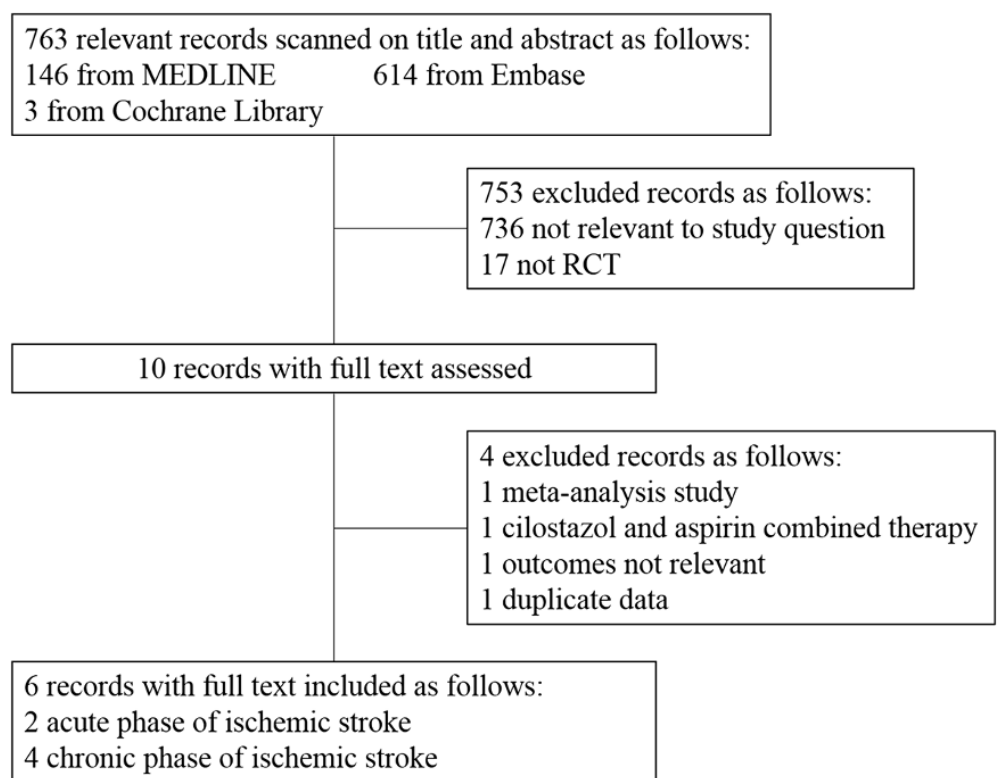

Figure 1 Study search, selection and inclusion process. 
Table 1 Characteristics of the included studies and outcome events

\begin{tabular}{|c|c|c|c|c|c|c|c|c|c|c|c|c|c|}
\hline Articles & Country & $\begin{array}{l}\text { Therapeutic } \\
\text { centre }\end{array}$ & Inclusion criteria & Drugs & Age & $\begin{array}{l}\text { Male } \\
\text { percentage (\%) }\end{array}$ & Dose & ITT population & Duration & ROCI \% & HSSH \% & $\begin{array}{l}\mathrm{mRS} \\
0-1 \%\end{array}$ & ACD \% \\
\hline \multirow[t]{2}{*}{ Gotoh 2000} & \multirow[t]{2}{*}{ Japan } & \multirow{2}{*}{$\begin{array}{l}183 \text { clinical } \\
\text { institutes }\end{array}$} & \multirow{2}{*}{$\begin{array}{l}\text { Cerebral infarction } \\
\text { at } 1 \text { to } 6 \text { months }\end{array}$} & Cilostazol & $65.2(\mathrm{NC})$ & 64.6 & 100 mg twice daily & $533 / 1067$ & \multirow[t]{2}{*}{$1-5$ years } & 5.7 & 0.8 & I & 1.7 \\
\hline & & & & Placebo & $65.1(\mathrm{NC})$ & 60.8 & NC & $534 / 1067$ & & 10.8 & 1.3 & / & 1.9 \\
\hline \multirow[t]{2}{*}{ Huang 2008} & \multirow[t]{2}{*}{ China } & \multirow{2}{*}{$\begin{array}{l}\text { Multiple } \\
\text { center trial }\end{array}$} & \multirow{2}{*}{$\begin{array}{l}\text { Cerebral infarction } \\
\text { at } 1 \text { to } 6 \text { months }\end{array}$} & Cilostazol & $60.14(10.05)$ & 66.9 & NC & $360 / 719$ & \multirow[t]{2}{*}{$1-1.5$ years } & 3.1 & 0.3 & / & 0.8 \\
\hline & & & & Aspirin & $60.31(9.71)$ & 70.5 & NC & $359 / 719$ & & 4.2 & 1.9 & / & 1.4 \\
\hline \multirow[t]{2}{*}{ Guo 2009} & \multirow[t]{2}{*}{ China } & \multirow{2}{*}{$\begin{array}{l}\text { Single } \\
\text { center trail }\end{array}$} & \multirow{2}{*}{$\begin{array}{l}\text { Cerebral infarction } \\
\text { at } 1 \text { to } 6 \text { months }\end{array}$} & Cilostazol & $59.44(10.63)$ & 35.3 & 100 mg twice daily & $34 / 68$ & \multirow[t]{2}{*}{1 year } & 5.9 & 0 & / & 0 \\
\hline & & & & Aspirin & $62.06(11.12)$ & 35.3 & 100 mgonce daily & $34 / 68$ & & 2.9 & 2.9 & / & 5.9 \\
\hline \multirow[t]{2}{*}{ Shinohara 2010} & \multirow[t]{2}{*}{ Japan } & \multirow{2}{*}{$\begin{array}{l}278 \text { clinical } \\
\text { institutes }\end{array}$} & \multirow{2}{*}{$\begin{array}{l}\text { Cerebral infarction } \\
\text { in the previous } \\
26 \text { weeks }\end{array}$} & Cilostazol & $63.5(9.2)$ & 71.7 & 100 mg twice daily & $1337 / 2672$ & \multirow[t]{2}{*}{$1-5$ years } & 5.4 & 0.7 & / & 1.0 \\
\hline & & & & Aspirin & $63.4(9.0)$ & 71.7 & 81 mg once daily & $1335 / 2672$ & & 6.6 & 2.3 & / & 1.0 \\
\hline \multirow[t]{2}{*}{ Lee 2011} & \multirow[t]{2}{*}{ Korea } & \multirow{2}{*}{$\begin{array}{l}12 \text { clinical } \\
\text { institutes }\end{array}$} & \multirow{2}{*}{$\begin{array}{l}\text { Cerebral infarction } \\
\text { within } 48 \mathrm{~h} \text { of onset }\end{array}$} & Cilostazol & $63(12)$ & 64.1 & 100 mg twice daily & $231 / 458$ & \multirow[t]{2}{*}{90 days } & 2.2 & 0 & 56.3 & 0.4 \\
\hline & & & & Aspirin & $63(12)$ & 58.6 & 300 mg/day & $227 / 458$ & & 4.0 & 0.9 & 56.8 & 0 \\
\hline \multirow[t]{2}{*}{ Shimizu 2013} & \multirow[t]{2}{*}{ Japan } & \multirow{2}{*}{$\begin{array}{l}55 \text { clinical } \\
\text { institutes }\end{array}$} & \multirow{2}{*}{$\begin{array}{l}\text { Cerebral infarction } \\
\text { within } 48 \mathrm{~h} \text { of onset }\end{array}$} & Cilostazol & $66.2(9.4)$ & 65.7 & 100 mg twice daily & $251 / 507$ & \multirow[t]{2}{*}{90 days } & 1.2 & 0.8 & 74.5 & 0 \\
\hline & & & & Placebo & 66.6 (8.9) & 68.4 & NC & $256 / 507$ & & 1.6 & 0.8 & 72.7 & 0 \\
\hline
\end{tabular}

ITT: intention to treat; ROCI: Recurrence of Cerebral Infarction; HSSH: Hemorrhage Stroke or Subarachnoid Hemorrhage; mRS: modifiedRankin Scale; ACD: All Case Death; NC: Not Clear. 
Table 2 Analysis and quality of the evidence using GRADE for efficacy and safety outcomes

\begin{tabular}{|c|c|c|c|c|c|c|c|c|}
\hline Outcomes & No of participants (studies) & Relative effect $(95 \% \mathrm{Cl})$ & Risk of bias & Inconsistency & Indirectness & Imprecision & Publication bias & Quality of the evidence (GRADE) \\
\hline \multicolumn{9}{|c|}{ 1. Subgroup Analysis - Cilostazol compared to Aspirin for the Secondary Prevention of Stroke in the Chronic phase } \\
\hline ROCI & 3459 (3 studies) & RR 0.82 (0.62 to 1.08$)$ & No serious & No serious & No serious & No serious & Undetected & $\oplus \oplus \oplus \oplus$ high \\
\hline HSSH & 3459 (3 studies) & RR 0.29 (0.15 to 0.56$)^{* * *}$ & No serious & No serious & No serious & No serious & Undetected & $\oplus \oplus \oplus \oplus$ high \\
\hline$A C D$ & 3459 (3 studies) & RR 0.80 (0.42 to 1.53 ) & No serious & No serious & No serious & No serious & Undetected & $\oplus \oplus \oplus \oplus$ high \\
\hline \multicolumn{9}{|c|}{ 2. Sensitivity Analysis - Cilostazol compared to Aspirin for the Secondary Prevention of Stroke in the Chronic phase without CSPS 2 trial } \\
\hline ROCI & 787 (2 studies) & RR 0.81 (0.40 to 1.66) & Serious $^{1}$ & No serious & No serious & No serious & Undetected & $\oplus \oplus \oplus \ominus$ moderate \\
\hline HSSH & 787 (2 studies) & RR $0.18(0.03 \text { to } 0.99)^{*}$ & Serious $^{1}$ & No serious & No serious & No serious & Undetected & $\oplus \oplus \oplus \ominus$ moderate \\
\hline ACD & 787 (2 studies) & RR 0.47 (0.13 to 1.64) & Serious $^{1}$ & No serious & No serious & No serious & Undetected & $\oplus \oplus \oplus \ominus$ moderate \\
\hline \multicolumn{9}{|c|}{ 3. Sensitivity Analysis - Cilostazol compared to Aspirin for the Secondary Prevention of Stroke in the Chronic phase without Guo-2009 trail } \\
\hline ROCI & 3391 (2 studies) & RR 0.80 (0.61 to 1.07) & No serious & No serious & No serious & No serious & Undetected & $\oplus \oplus \oplus \oplus$ high \\
\hline HSSH & 3391 (2 studies) & RR $0.29(0.15 \text { to } 0.56)^{* * *}$ & No serious & No serious & No serious & No serious & Undetected & $\oplus \oplus \oplus \oplus$ high \\
\hline ACD & 3391 (2 studies) & RR 0.89 (0.45 to 1.73 ) & No serious & No serious & No serious & No serious & Undetected & $\oplus \oplus \oplus \oplus \mathbf{h i g h}$ \\
\hline
\end{tabular}

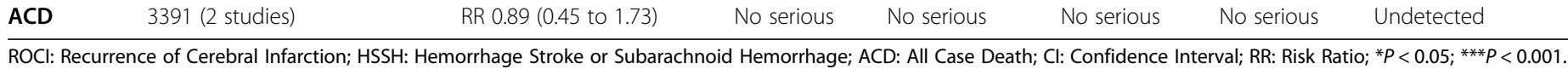

ROCl: Recurrence of Cerebral Infarction; $\mathrm{HSSH}$ :
1Potential bias because of unclear of blinding GRADE Working Group grades of evidence:

High quality: Further research is very unlikely to change our confidence in the estimate of effect.

Moderate quality: Further research is likely to have an important impact on our confidence in the estimate of effect and may change the estimate.

Low quality: Further research is very likely to have an important impact on our confidence in the estimate of effect and is likely to change the estimate.

Very low quality: We are very uncertain about the estimate. 
(RR 0.57, 95\% CI 0.17 to $1.94, p=0.37$ ) and ACD (RR $0.90,95 \%$ CI 0.37 to $2.20, p=0.82$ ). These data were not shown in the tables or figures. In the aspirin-controlled studies, cilostazol therapy was associated with an insignificant $18 \%$ reduction in the ROCI (RR $0.82,95 \%$ CI 0.62 to $1.08, p=0.15$; Figure $2 \mathrm{~A}$ ), and a $71 \%$ reduction in the HSSH (RR $0.29,95 \%$ CI 0.15 to $0.56, p=0.0002$; Figure 2B) with no significant difference in the ACD (RR $0.80,95 \%$ CI 0.42 to $1.53, p=0.51$; Figure 2 C). In order to detect whether the consolidated results were influenced by one study with a large population [9], we performed the sensitivity analysis to confirm that the results were stable (Table 2).

\section{The efficacy and safety of cilostazol in acute phase}

For the analysis of the efficacy and safety of cilostazol in acute phase, 965 patients from 2 studies were included (507 patients from 1 study randomized to cilostazol or placebo, and 458 patients from 1 study to cilostazol or aspirin). In the placebo-controlled study, cilostazol therapy showed no significant efficacy in the ROCI (RR 0.76, 95\% CI 0.17 to $3.38, p=0.72$ ), and a similar result in the HSSH (RR $1.02,95 \%$ CI 0.14 to $7.18, p=0.98$ ). In the aspirincontrolled study, cilostazol therapy was associated with no significant efficacy in the ROCI (RR 0.55, 95\% CI 0.19 to 1.60, $p=0.27$ ), and showed the similar incidence in the HSSH (RR 0.20, 95\% CI 0.01 to $4.12, p=0.30$ ) and ACD (RR 2.95, 95\% CI 0.12 to $72, p=0.51$ ). No significant difference existed between cilostazol therapy and placebo or aspirin groups in the mRS (data not shown).

\section{Risk of the bias}

For allocation concealment, risk of bias was high in one study [16] and unclear in another one [4]. For blinding of participants and personnel, risk of bias was high in one study [16]. For blinding of outcomes assessment, risk of bias was high in one study [16]. Except these four items, no high risk of bias was observed in any of the other items (Figure 3).

\section{Discussion}

The present systematic review and meta-analysis, including 6 RCTs in 5491 patients, evaluated the efficacy and acceptability of cilostazol for the secondary prevention of ischemic stroke. Our results suggest that cilostazol therapy leads to a significant reduction in ROCI compared to placebo, and a lower incidence of the HSSH compared with aspirin in the chronic phase. While in acute phase,
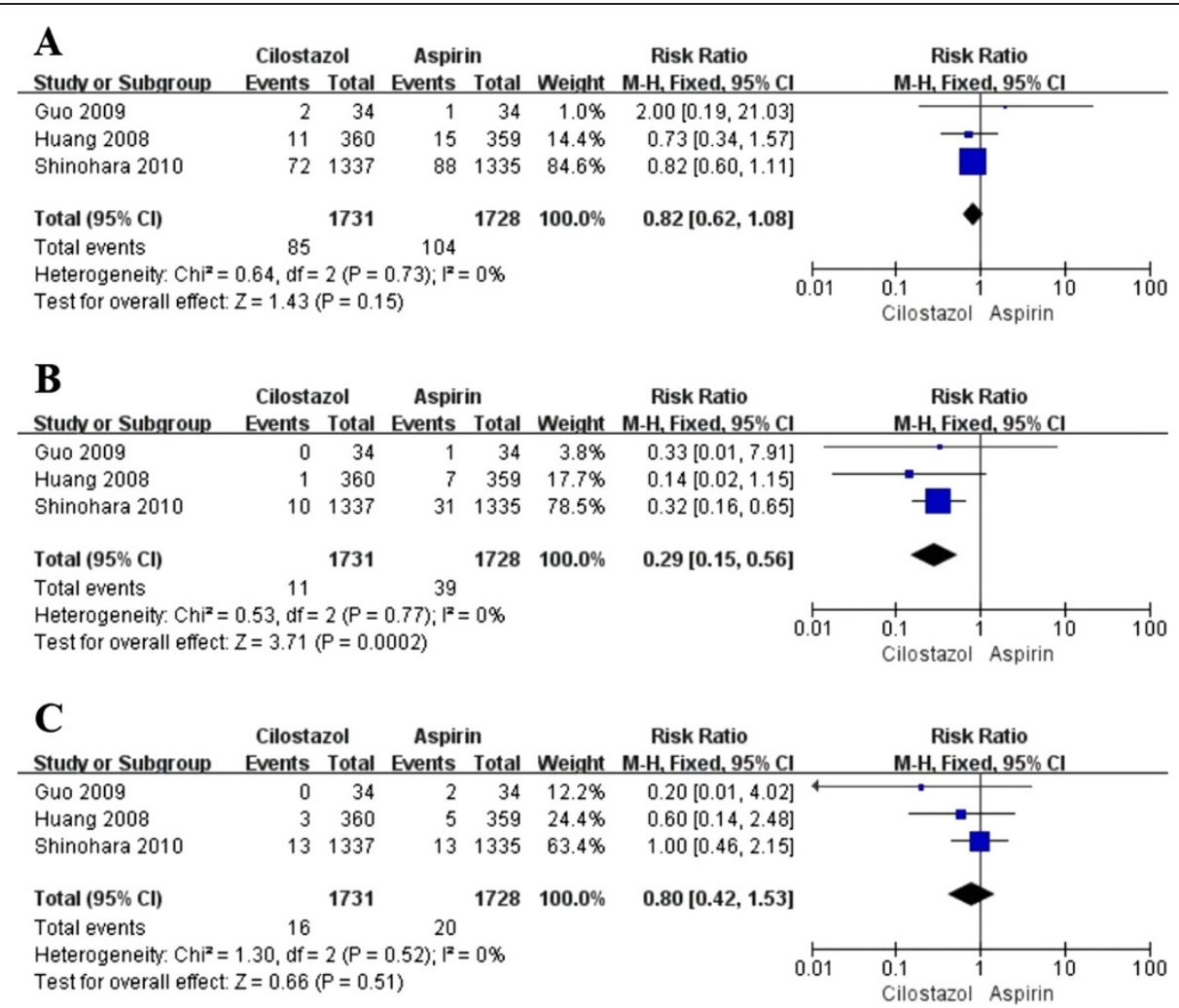

Figure 2 Pooled relative risk of stroke recurrence,hemorrhagic stroke and all cause death between cilosatzol and aspirin groups insecondary prevention of stroke. (A) Pooled relative risk estimates on recurrence of cerebral infarction. (B) Pooled relative risk estimates on hemorrhage stroke or subarachnoid hemorrhage. (C) Pooled relative risk estimates on all cause death. The diamond indicates the estimated relative risk (95\% confidence interval) for all patients together. 


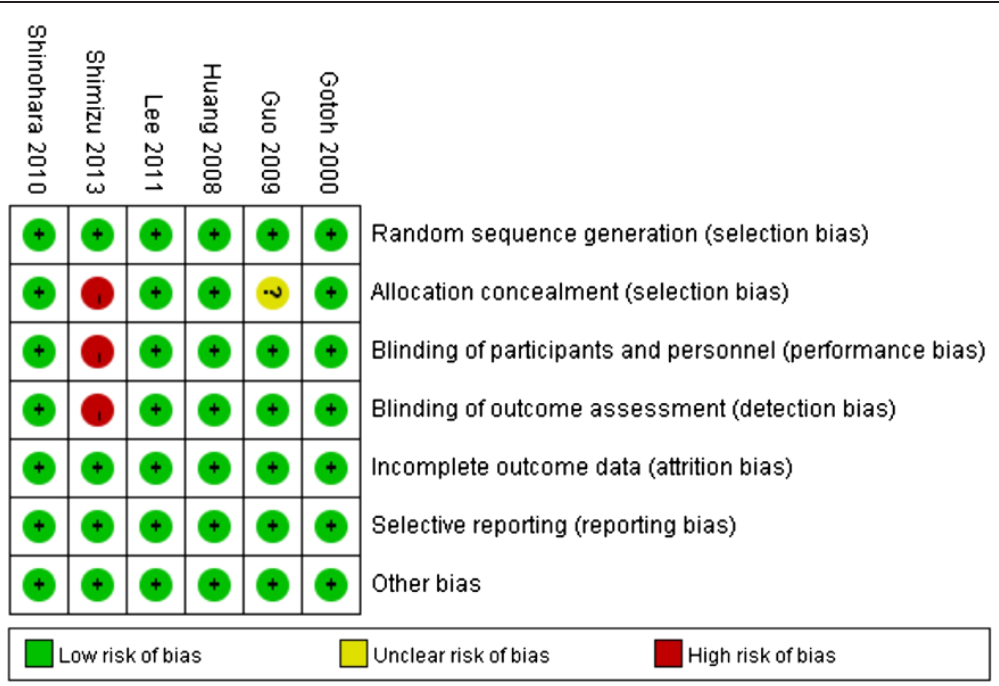

Figure 3 Risk of bias: a summary table for each risk of bias items for each study.

cilostazol showed no better efficacy in the ROCI than placebo, and had a similar incidence of the HSSH to aspirin therapy. These findings support that cilostazol may be an advisable therapeutic alternative for aspirin in the secondary prevention of the chronic phase of ischemic stroke. Quality of the evidence for the outcomes from chronic phase studies assessed by GRADE was high or moderate, which provides a sufficient confidence at the application of cilostazol in clinical practice.

Compared with the methodology of the previous metaanalysis $[11,12]$, the present systematic review explored the scope of cilostazol application for the secondary prevention of ischemic stroke. First, we defined the acute and chronic phases of ischemic stroke according to the onset-totreatment time as follows: i) acute phase refers to patients having had an ischemic stroke within the preceding 48 hours; ii) the definition of chronic phase refers that patients were enrolled 1 to 6 months after ischemic stroke. The previous meta-analysis [11,12], did not distinguish between the acute and chronic phases. The present systematic review showed that cilostazol had controversial efficacy in the prevention of acute ischemic stroke. It is mainly due to the starting treatment time after onset. Many of the previous studies have reported that approximately $20 \%-37 \%$ of patients with acute ischemic stroke worsened gradually or stepwise after onset [21,22]. Among these patients, 58\%$82 \%$ deteriorated progressively during the first 24 hours [23-25]. Hence, patients included within the first 24 hours of the Shimizu et al. [10] study may have progressed to a worse condition, which influenced the efficacy of cilostazol. In cases relating to thechronic phase of ischemic stroke, cilostazol showed a significant beneficial effect, which was consistent with the previous studies $[2,4,9,11,12]$. Second, in the previous meta-analysis [11,12], only aspirincontrolled RCTs were included to assess the efficacy of the cilostazol, which concluded that no significant difference existed in ROCI between cilostazol and aspirin. In the present systematic review, we have included both aspirin and placebo-controlled RCTs, which resulted in a more comprehensive conclusion that cilostazol has a definite effect in the ROCI, but not better than aspirin. Furthermore, we concluded that cilostazol is safer than aspirin in the HSSH, which was similar to the placebo. Third, we used a fixed-effects approach in meta-analysis where data did not indicate heterogeneity.

Current stroke guidelines from the American Stroke Association (ASA) and American Heart Association (AHA), recommend aspirin, clopidogrel, or aspirin plus extended-release dipyridamole as first-line options for secondary prevention of ischemic events (Class IIa, Level of Evidence A) [26]. Aspirin has a wide dose range from 50 to $1300 \mathrm{mg} / \mathrm{d}$ to prevent the reoccurrence of stroke [27]. However, both high- or low-dose aspirin may cause intracranial hemorrhagic events, which could limit its clinical application [28]. Cilostazol, a novel antiplatelet drug, prevents the recurrence of ischemic stroke through its antiplatelet effects [8], vasodilation, inhibition of vascular smooth muscle cell growth, and neuroprotection. Several randomized, multicentered trials demonstrated the preventable effect of cilostazol in patients with a previous stroke. According to the Cilostazol Stroke Prevention Study, patients with a prior stroke, who were allocated to cilostazol $100 \mathrm{mg}$ twice daily or placebo, showed that cilostazol therapy reached a significant $58.3 \%$ reduction in ROCI, with no clinically significant adverse reactions [15]. Three other RCTs compared with aspirin show that cilostazol not only had similar therapeutic effects with aspirin in the ROCI, but also had a significant reduction in the HSSH $[2,4,9]$. In the animal studies, cilostazol showed a better effect than aspirin in the reduction of brain damage after 
ischemic stroke though suppressing disruption of the microvasculature and increasing the residual perfusion of microcirculation $[29,30]$. A pilot study, reported that patients treated with combined therapy had less neurological deterioration and a more favorable functional status than those treated with aspirin alone in secondary prevention of acute ischemic stroke [31]. Another double-blind multicenter trial containing 244 aspirin subjects with ischemic stroke who were randomly assigned to receive cilostazol $100 \mathrm{mg}$ twice daily or placebo, observed a trend toward enhanced antiplatelet effects when cilostazol was added to aspirin in ischemic stroke patients [32]. It should be noted however, that cilostazol was more likely to cause several adverse events other than intracranial hematoma in comparison with aspirin [9]. The most common adverse events were put in descending order of occurrence as follows: headache, diarrhea, palpitations, dizziness, and tachycardia [9]. These findings supported that cilostazol is an alternative drug of aspirin but still needs large, randomized, multicentered trials to confirm the efficacy and safety of cilostazol.

In the present review, several factors may affect the combining of data, despite the statistics showing a low heterogeneity. For the treatment duration, 2 studies $[9,15]$ followed up for 1-5 years, while another two studies $[2,4]$ were 1-1.5 years. However, this difference in the date of treatment may only play a minor role because the KaplanMeier curves for the accumulation of primary endpoints showed a steady trend after 400 days [2]. For the stroke etiologies, the most common type was lacunar infarction followed by atherothrombotic infarction in the included studies. Three studies provided similar findings in about $65 \%-75 \%$ proportion of lacunar infarction, and no significant difference existed between cilostazol and controlled groups $[9,15,16]$. Another three studies lacked data regarding stroke etiologies $[2,4,10]$, which might affect the combining of data to some degree. For the different vascular risk factors, a previous review has indicated that hypertension, diebetes, and hyperlipidemia maybe the main causes to influence the efficacy of secondary prevention [33]. In the present review, all six included studies recruited approximately $70 \%$ hypertension, 30\% diebetes, and 30\% hyperlipidemia. Only one study showed significant differences in systolic blood pressure between cliostazol and controlled groups [9]. Although the authors clarified that no interaction existed between treatment group and measurement time-points for systolic or diastolic blood pressure, the results of sensitivity analysis, without this study, showed that the difference became smaller but did not reverse the results (Table 2).

Several limitations of the present study should be considered. First, the present meta-analysis only included 4 studies for the chronic phase of ischemic stroke, and 2 studies for acute stroke. The number of studies is small, which may cause reporting bias. The result of secondary prevention for chronic phase of ischemic stroke was based on one placebo-controlled RCT, which was not an effect size. This same limitation also existed in the results of acute phase. Caution should be used when applying these results in the clinical setting. Secondly, not all of the included studies were double-blind, randomized, controlled trails. The Cilostazol for the Prevention of Acute Progressing Stroke was an open, multicenter, randomized controlled trial [16]. The information of blinding was not available from the study of Guo et al. [4], which made it difficult to determine if this trial was double-blinded. Finally, all of the patients from the included studies were of Asian descent, suggesting a limited confidence when applying this data to other populations. While aspirin was an ideal option for the secondary prevention of stroke in western countries, it did not seem suitable for those of Asian descent [5]. Previous studies have reported that Asians are at a higher risk forside effects including recurrent ischemic and hemorrhagic strokes in the secondary stroke prevention phase [5]. Genetic factors in different race-ethnicities, as independent predictors of cerebrovascular disease, maybe the main reason for high risk of side effects in Asian population [34-36]. In addition, higher and poorer control of blood pressure in Asians may be another factor contributing to the poor efficacy of aspirin in secondary stroke prevention [5]. Hence, cilostazol might be a safer option for Asians because of its reduced risk of intracerebral hemorrhage when compared to aspirin. All of these limitations were considered in the evaluating the quality of evidence.

\section{Conclusion}

Cilostazol therapy played a crucial role in the secondary prevention of ischemic stroke in chronic phase. No significant difference was presented in ROCI between cilostazol and aspirin, but cilostazol was deemed to be safer. Prospective large RCTs will provide more evidence for cilostazol as an alternative drug for aspirin in secondary prevention of stroke.

\section{Appendix}

1. (cilostazol [Title/Abstract] OR pletal [Title/Abstract] OR pletaal[Title/Abstract] OR OPC 13013 [Title/ Abstract] OR OPC 21 [Title/Abstract])

2. (aspirin [Title/Abstract] OR acetylsalicylic acid [Title/ Abstract] OR acetyl salicylic acid[Title/Abstract] OR acetosalicylic acid [Title/Abstract] OR placebo [Title/ Abstract])

3. (ischemi" [Title/Abstract] OR stroke [Title/Abstract] OR cerebrovascular [Title/Abstract] OR intracerebral [Title/Abstract] OR embolism [Title/Abstract] OR thrombosis [Title/Abstract])

4. 1 AND 2 AND 3

5. Limit 4 to humans 


\section{Competing interests}

The authors declare that they have no competing interests.

\section{Authors' contributions}

$J M Z$ is the principal investigator. SC designed the study and developed the analysis plan. LGS and JLP analyzed the data and performed meta-analysis. LGS contributed in writing of the article. LX and JM revised the manuscript and polish the language. All authors read and approved the final manuscript.

\section{Acknowledgements}

This study was supported by National Natural Science Foundation of China (No.81171096 and No. 81371433) to JM Zhang.

\section{Author details}

${ }^{1}$ Department of Neurosurgery, Second Affiliated Hospital, School of Medicine, Zhejiang University, 88 Jiefang Road, Hangzhou 310009, Zhejiang, China. ${ }^{2}$ Department of Neurology, Second Affiliated Hospital, School of Medicine, Zhejiang University, Hangzhou, Zhejiang, China. ${ }^{3}$ Department of Physiology and Pharmacology, Loma Linda University, Loma Linda, CA, USA.

Received: 20 July 2014 Accepted: 11 December 2014

Published online: 20 December 2014

\section{References}

1. Murray CJ, Lopez AD: Global mortality, disability, and the contribution of risk factors: global burden of disease study. Lancet 1997, 349:1436-1442.

2. Huang $Y$, Cheng $Y$, Wu J, Li Y, Xu E, Hong Z, Li Z, Zhang W, Ding M, Gao X, Fan D, Zeng J, Wong K, Lu C, Xiao J, Yao C: Cilostazol as an alternative to aspirin after ischaemic stroke: a randomised, double-blind, pilot study. Lancet Neurol 2008, 7:494-499.

3. Pendlebury ST, Rothwell PM: Prevalence, incidence, and factors associated with pre-stroke and post-stroke dementia: a systematic review and meta-analysis. Lancet Neurol 2009, 8:1006-1018.

4. Guo JJ, Xu E, Lin QY, Zeng GL, Xie HF: Effect of cilostazol on cerebral arteries in secondary prevention of ischemic stroke. Neurosci Bull 2009, 25:383-390.

5. Estol CJ, Bath PM, Gorelick PB, Cotton D, Martin RH, Committee PRP, Investigators PR: Differences in ischemic and hemorrhagic recurrence rates among race-ethnic groups in the PRoFESS secondary stroke prevention trial. Int J Stroke 2014, Suppl A100:43-47.

6. He J, Whelton PK, Vu B, Klag MJ: Aspirin and risk of hemorrhagic stroke: a meta-analysis of randomized controlled trials. JAMA 1998, 280:1930-1935.

7. Jaspers Focks J, Tielemans MM, van Rossum LG, Eikendal T, Brouwer MA, Jansen JB, Laheij RJ, Verheugt FW, van Oijen MG: Gastrointestinal symptoms in low-dose aspirin users: a comparison between plain and buffered aspirin. Neth Heart J 2014, 22:107-112.

8. Sudo T, Tachibana K, Toga K, Tochizawa S, Inoue Y, Kimura Y, Hidaka H: Potent effects of novel anti-platelet aggregatory cilostamide analogues on recombinant cyclic nucleotide phosphodiesterase isozyme activity. Biochem Pharmacol 2000, 59:347-356.

9. Shinohara $Y$, Katayama Y, Uchiyama S, Yamaguchi T, Handa S, Matsuoka K, Ohashi Y, Tanahashi N, Yamamoto H, Genka C, Kitagawa Y, Kusuoka H, Nishimaru K, Tsushima M, Koretsune Y, Sawada T, Hamada C: Cilostazol for prevention of secondary stroke (CSPS 2): an aspirin-controlled, doubleblind, randomised non-inferiority trial. Lancet Neurol 2010, 9:959-968.

10. Lee YS, Bae HJ, Kang DW, Lee SH, Yu K, Park JM, Cho YJ, Hong KS, Kim DE, Kwon SU, Lee KB, Rha JH, Koo J, Han MG, Lee SJ, Lee JH, Jung SW, Lee BC, Kim JS: Cilostazol in Acute Ischemic Stroke Treatment (CAIST Trial): a randomized double-blind non-inferiority trial. Cerebrovasc Dis 2011, 32:65-71.

11. Dinicolantonio JJ, Lavie CJ, Fares H, Menezes AR, O'Keefe JH, Bangalore S, Messerli FH: Meta-analysis of cilostazol versus aspirin for the secondary prevention of stroke. Am J Cardiol 2013, 112:1230-1234.

12. Qian Y, Bi Q: Systematic study of cilostazol on secondary stroke prevention: a meta-analysis. Eur J Med Res 2013, 18:53.

13. Buras JA, Reenstra WR: Endothelial-neutrophil interactions during ischemia and reperfusion injury: basic mechanisms of hyperbaric oxygen. Neurol Res 2007, 29:127-131.

14. Caplan LR: Worsening in ischemic stroke patients: is it time for a new strategy? Stroke 2002, 33:1443-1445.
15. Gotoh F, Tohgi H, Hirai S, Terashi A, Fukuuchi Y, Otomo E, Shinohara Y, Itoh E, Matsuda T, Sawada T, Yamaguchi T, Nishimaru K, Ohashi Y: Cilostazol stroke prevention study: a placebo-controlled double-blind trial for secondary prevention of cerebral infarction. J Stroke Cerebrovasc Dis 2000, 9:147-157.

16. Shimizu H, Tominaga T, Ogawa A, Kayama T, Mizoi K, Saito K, Terayama Y, Ogasawara K, Mori E: Cilostazol for the prevention of acute progressing stroke: a multicenter, randomized controlled trial.

J Stroke Cerebrovasc Dis 2013, 22:449-456.

17. Brozek JL, Akl EA, Alonso-Coello P, Lang D, Jaeschke R, Williams JW, Phillips B, Lelgemann M, Lethaby A, Bousquet J, Guyatt GH, Schunemann HJ, Group GW: Grading quality of evidence and strength of recommendations in clinical practice guidelines. Part 1 of 3 . An overview of the GRADE approach and grading quality of evidence about interventions. Allergy 2009, 64:669-677.

18. Brozek JL, Akl EA, Jaeschke R, Lang DM, Bossuyt $P$, Glasziou P, Helfand M, Ueffing E, Alonso-Coello P, Meerpohl J, Phillips B, Horvath AR, Bousquet J, Guyatt GH, Schunemann HJ: Grading quality of evidence and strength of recommendations in clinical practice guidelines: Part 2 of 3. The GRADE approach to grading quality of evidence about diagnostic tests and strategies. Allergy 2009, 64:1109-1116.

19. Brozek JL, Akl EA, Compalati E, Kreis J, Terracciano L, Fiocchi A, Ueffing E, Andrews J, Alonso-Coello P, Meerpohl JJ, Lang DM, Jaeschke R, Williams JW, Jr., Phillips B, Lethaby A, Bossuyt P, Glasziou P, Helfand M, Watine J, Afilalo M, Welch V, Montedori A, Abraha I, Horvath AR, Bousquet J, Guyatt GH, Schunemann HJ: Grading quality of evidence and strength of recommendations in clinical practice guidelines part 3 of 3 . The GRADE approach to developing recommendations. Allergy 2011, 66:588-595.

20. Liberati A, Altman DG, Tetzlaff J, Mulrow C, Gotzsche PC, loannidis JP, Clarke M, Devereaux PJ, Kleijnen J, Moher D: The PRISMA statement for reporting systematic reviews and meta-analyses of studies that evaluate healthcare interventions: explanation and elaboration. BMJ 2009, 339:b2700.

21. Mohr JP, Caplan LR, Melski JW, Goldstein RJ, Duncan GW, Kistler JP, Pessin MS, Bleich HL: The Harvard Cooperative Stroke Registry: a prospective registry. Neurology 1978, 28:754-762.

22. Marti-Vilalta JL, Arboix A: The Barcelona stroke registry. Eur Neurol 1999, 41:135-142.

23. Yamamoto H, Bogousslavsky J, van Melle G: Different predictors of neurological worsening in different causes of stroke. Arch Neurol 1998, 55:481-486

24. Arboix A, Marti-Vilalta JL, Garcia JH: Clinical study of 227 patients with lacunar infarcts. Stroke 1990, 21:842-847.

25. Norrving B, Cronqvist S: Clinical and radiologic features of lacunar versus nonlacunar minor stroke. Stroke 1989, 20:59-64

26. Sacco RL, Adams R, Albers G, Alberts MJ, Benavente O, Furie K, Goldstein LB, Gorelick P, Halperin J, Harbaugh R, Johnston SC, Katzan I, Kelly-Hayes M, Kenton EJ, Marks M, Schwamm LH, Tomsick T: Guidelines for prevention of stroke in patients with ischemic stroke or transient ischemic attack: a statement for healthcare professionals from the American Heart Association/American Stroke Association Council on Stroke: co-sponsored by the Council on Cardiovascular Radiology and Intervention: the American Academy of Neurology affirms the value of this guideline. Circulation 2006, 113:e409-e449.

27. Diener HC, Cunha L, Forbes C, Sivenius J, Smets P, Lowenthal A: European Stroke Prevention Study. 2. Dipyridamole and acetylsalicylic acid in the secondary prevention of stroke. J Neurol Sci 1996, 143:1-13.

28. Hansson L, Zanchetti A, Carruthers SG, Dahlof B, Elmfeldt D, Julius S, Menard J, Rahn $\mathrm{KH}$, Wedel $\mathrm{H}$, Westerling S: Effects of intensive blood-pressure lowering and low-dose aspirin in patients with hypertension: principal results of the Hypertension Optimal Treatment (HOT) randomised trial. HOT Study Group. Lancet 1998, 351:1755-1762.

29. Kasahara Y, Nakagomi T, Matsuyama T, Stern D, Taguchi A: Cilostazol reduces the risk of hemorrhagic infarction after administration of tissue-type plasminogen activator in a murine stroke model. Stroke 2012, 43:499-506.

30. Oyama N, Yagita Y, Kawamura M, Sugiyama Y, Terasaki Y, Omura-Matsuoka E, Sasaki T, Kitagawa K: Cilostazol, not aspirin, reduces ischemic brain injury via endothelial protection in spontaneously hypertensive rats. Stroke 2011, 42:2571-2577.

31. Nakamura T, Tsuruta S, Uchiyama S: Cilostazol combined with aspirin prevents early neurological deterioration in patients with acute ischemic stroke: a pilot study. J Neurol Sci 2012, 313:22-26. 
32. Lee JH, Cha JK, Lee SJ, Ha SW, Kwon SU: Addition of cilostazol reduces biological aspirin resistance in aspirin users with ischaemic stroke: a double-blind randomized clinical trial. Eur J Neurol 2010, 17:434-442.

33. Paciaroni M, Bogousslavsky J: Primary and secondary prevention of ischemic stroke. Eur Neurol 2010, 63:267-278.

34. Feldmann E, Daneault N, Kwan E, Ho KJ, Pessin MS, Langenberg P, Caplan LR: Chinese-white differences in the distribution of occlusive cerebrovascular disease. Neurology 1990, 40:1541-1545.

35. Inzitari D, Hachinski VC, Taylor DW, Barnett HJ: Racial differences in the anterior circulation in cerebrovascular disease. How much can be explained by risk factors? Arch Neurol 1990, 47:1080-1084.

36. Waddy SP, Cotsonis G, Lynn MJ, Frankel MR, Chaturvedi S, Williams JE, Chimowitz M: Racial differences in vascular risk factors and outcomes of patients with intracranial atherosclerotic arterial stenosis. Stroke 2009, 40:719-725.

\section{Submit your next manuscript to BioMed Central and take full advantage of:}

- Convenient online submission

- Thorough peer review

- No space constraints or color figure charges

- Immediate publication on acceptance

- Inclusion in PubMed, CAS, Scopus and Google Scholar

- Research which is freely available for redistribution 